\title{
Morphological Detection based on Size and Contrast Criteria Application to Cells Detection
}

\author{
P. Salembier, A. Gasull, F. Marques, E. Sayrol \\ Department of Signal Theory and Communications \\ Universitat Politecnica de Catalunya \\ ETSET, Apdo. 30.002, 08080 Barcelona, Spain
}

\begin{abstract}
This paper deals with a detection algorithm relying on size and contrast criteria. It is suitable for a large range of applications where $a$ priori information about the size and the contrast of the objects to detect is available. The detection is performed in three separate steps: the first one is a preprocessing which removes unuseful information with a size criterion. The second one performs a feature extraction based on contrast. Finally, the last step is the decision itself. All these steps make use of morphological transformations because of their ability to deal with the criteria of interest and of their low computational cost. As an example, this algorithm is applied to the automatic detection of spermatozoa.
\end{abstract}

\section{INTRODUCTION}

A large number of visual detection problems are very easily and efficiently solved by humans which make use of rather high level criteria. Example of such criteria are the shape, the size, the contrast or the connectivity of the various image components or objects. Mathematical morphology [1] is increasingly used for practical problems such as automatic detection. One of the causes of this success is its ability to deal with intuitive but high level notions such those mentioned above. Moreover, morphological transformations are based on nonlinear operations such as minimum and maximum which are well suited to image processing because most of the objects in a scene are opaque and perception does not follow the linear superposition property. Finally, morphological operations can be implemented in very efficient ways which make them attractive for practical applications.

In almost any detection process three steps can be distinguished: preprocessing, feature extraction and decision. The goal of the preprocessing is to remove unuseful information and therefore to simplify the original image. The feature extraction computes the pertinent parameter(s) which characterise(s) the elements to detect. Finally, the result is given by a decision step. The goal of this paper is to present a morphological detection algorithm where the preprocessing relies on a size criterion and the feature extraction on contrast. This type of algorithms has proved its usefulness for automatic visual inspection in industrial environment [2] and it is believed that it is suitable for biomedical applications where detections have to be performed following size and/or contrast criteria. As an example, this algorithm is applied to the unsupervised detection of spermatozoa in semen but it can be used for a larger class of detection problems. In this specific application, the detection is the first step towards an automatic analysis of semen including counting and motion analysis [3].

\section{0-7803-0785-2/92\$03.00 @IEEE}

The organisation of this paper is as follows: the next section describes briefly the main features and characteristics of the detection algorithm. Section 3 is devoted to the description of a particular example of detection and to the discussion of the results.

\section{DETECTION ALGORITHM}

As discussed in the introduction, the detection algorithm is composed of three fundamental steps: preprocessing, feature extraction and decision. The definition of each step depends on the particular application and, in order to get a simple and robust detection scheme, as much a priori information as possible should be used. In our practical detection problem, images such as the one shown in Fig. 1a should be processed. The objects of interest appear as medium-sized bright spots with high to moderate contrast with respect to their surroundings. The other image components can be viewed as detection noise. They are mainly composed of large bright spots, dark elements of all sizes, acquisition noise which are very small points of both polarity and in some cases variations of the average grey level value. As can be seen, this analysis leads to a detection relying on size, contrast and polarity criteria. The detection problem can be defined as the search of medium-sized objects with bright contrast. This definition leads us rather naturally to the following steps:

\section{A Preprocessing: Size-sensitive multiresolution decomposition}

The preprocessing should simplify the original image by removing objects that are not possible cells of interest. In our case, it removes all very small components (noise), all large components which obviously are not cells of interest and finally all dark objects. It is based on a three level sizesensitive multiresolution decomposition [4]. As illustrated in Fig. 2, it decomposes the original image in three, respectively composed of small bright, medium-sized bright, dark and large bright components. It is based on two opening filters with reconstruction (due to space limitation the reader is referred to [4] for more details). For further processing, only the image composed of medium-sized bright objects is kept.

B Feature extraction: Contrast enhancement

After the preprocessing, the image is quite simple and the feature extraction can be performed with a simple contrast enhancement technique (See Fig. 2). We use the following one [5]:

$$
g=f-\operatorname{MIN}\{\operatorname{open}(\operatorname{close}(f)), f\}
$$

where $f$ and $g$ respectively denote the original and resulting images. This very simple contrast enhancement technique can be viewed has a kind of improved Top Hat transform which compensates for detection noise (see [5] for more details).

\section{Decision}

After preprocessing and feature extraction, the resulting image is very simple and the decision can be taken with extremely 
simple techniques such as thresholding followed, if necessary, by a "cleaning" step which will remove obvious detection errors. In our case and with the set of images we had, this "cleaning" step was not necessary.

\section{APPLICATION TO CELLS DETECTION}

Let us describe an example of detection. The original image is shown on Fig. 1a. It is first simplified as illustrated in Fig. 1b. This result was achieved with two opening with squared structuring elements of size $3 * 3$ and $11 * 11$ and geodesic reconstruction (8 connectivity) [4]. As can be seen the multiresolution decomposition is extremely efficient for this simplification step. The image after contrast enhancement is presented in Fig 1c. In this last case, a structuring element of size $7 * 7$ was used. Finally, the detection result is shown in Fig. 1d. One can see that the spermatozoa are successfully detected even those with a low contrast. Finally, let us mention that the morphological transformations involved in this detection algorithm can be implemented in very efficient ways. For example, the whole processing time required for a $512 * 512$ image on a $\mathrm{PC}$ is of the order of a few seconds which makes it suitable for practical applications.

\section{REFERENCES}

[1] J. Serra, Image Analysis and Mathematical Morphology, Academic Press, Vol. 1 (1982), Vol. 2 (1988).

[2] P. Salembier, Multiscale Image Analysis and Modeling using Rank Order Based Filters - Application to Defect Detection -, PhD Dissertation No937, EPFL, Lausanne, June 1991.

[3] E. Sayrol, A. Gasull, Procesado Digital de Imagenes para la Detección y Seguimiento de Celulas, Proc. of URSI Conf., Santander, Spain, 25-27 Sept. 1989.

[4] P. Salembier, M. Kunt, Size-sensitive Multiresolution Decomposition of images using rank order based filters, Signal Processing, Vol. 27, No 2, May 1992.
[5] P. Salembier, Comparison of soine morphological segmentation algorithms based on contrast enhancement Application to automatic defect detection -, in SIGNAL PROCESSING V: Theories and Applications, Torres, Masgrau, Lagunas (eds), Elsevier Science Pub. 90 (EUSIPCO 90), pp.833-836. a

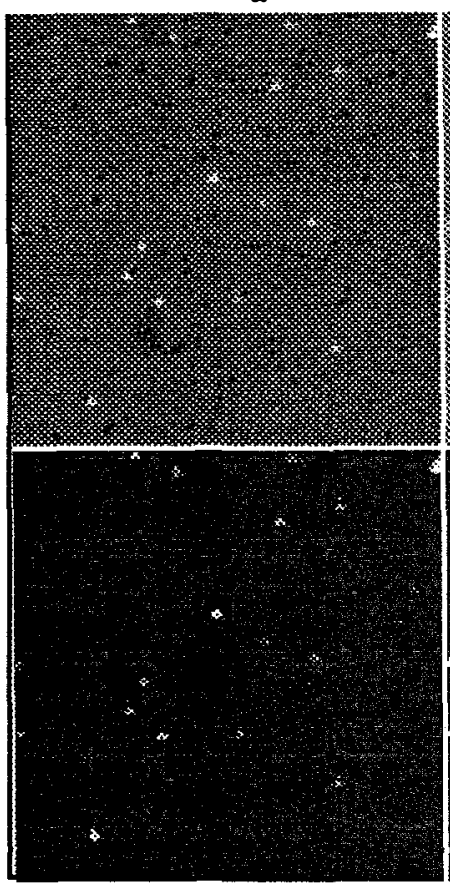

C b

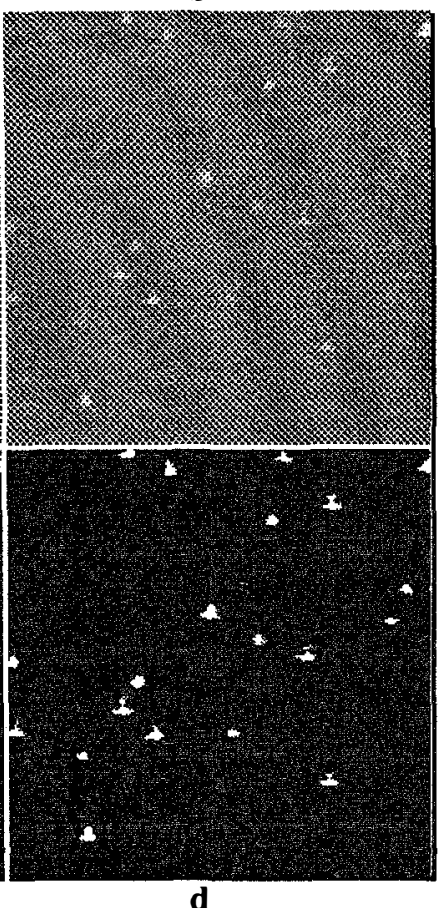

d
Fig.1: Example of detection

a: Original image

b: Simplified image after multiresolution decomposition

c: Result of contrast enhancement d: Final detection result

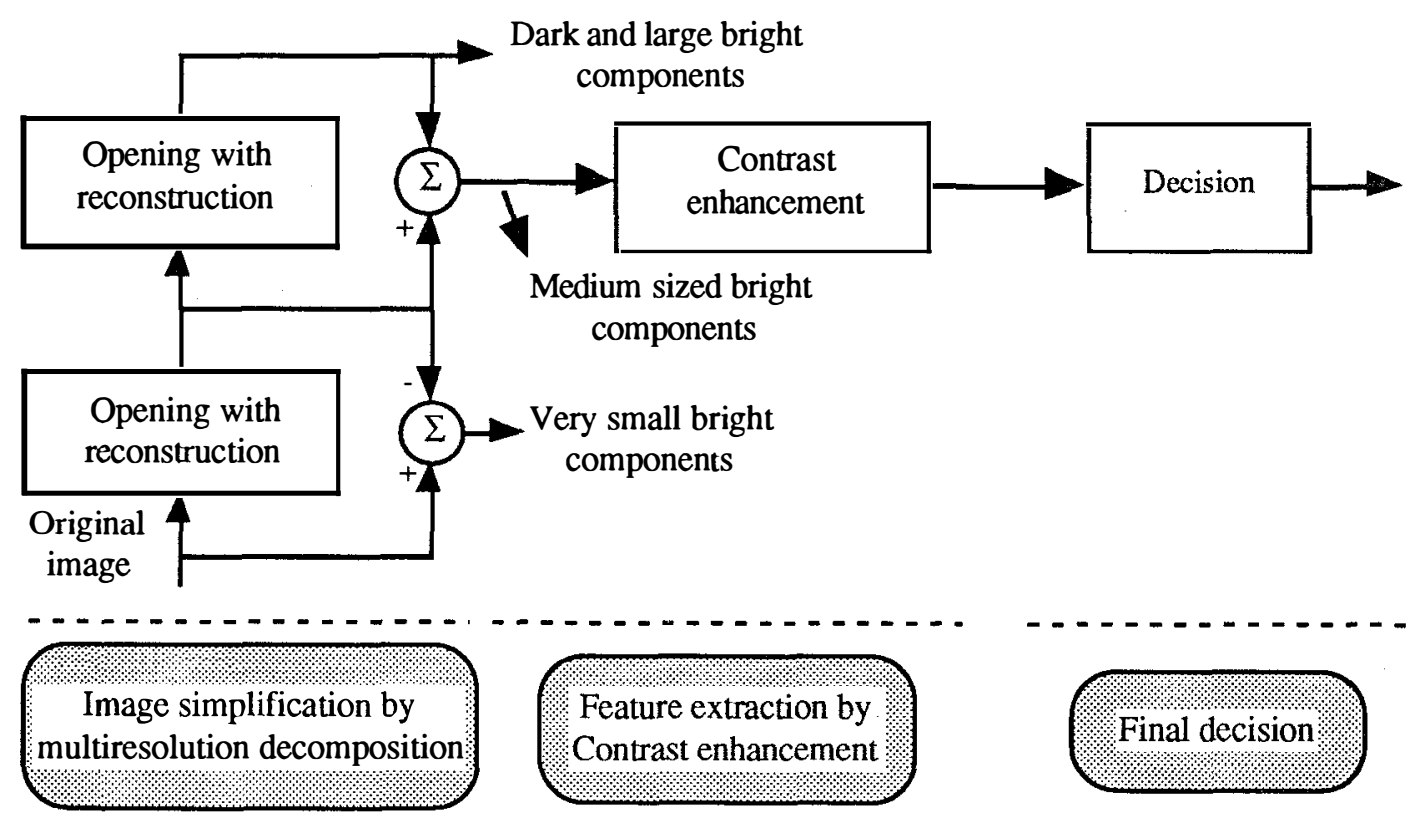

Fig. 2: Morphological detection scheme 